HEP/123-qed

\title{
Mechanism of carrier-induced ferromagnetism in magnetic semiconductors
}

\author{
Masao Takahashi \\ Kanagawa Institute of Technology \\ 1030 Shimo-Ogino, Atsugi-shi 243-0292, Japan \\ Kenn Kubo \\ Department of Physics, Aoyama Gakuin University \\ Setagaya, Tokyo 157-8572, Japan
}

(October 30, 2018)

\begin{abstract}
Taking into account both random impurity distribution and thermal fluctuations of localized spins, we have performed a model calculation for the carrier (hole) state in $\mathrm{Ga}_{1-x} \mathrm{Mn}_{x}$ As by using the coherent potential approximation (CPA). The result reveals that a $p$-hole in the band tail of $\mathrm{Ga}_{1-x} \mathrm{Mn}_{x}$ As is not like a free carrier but is rather virtually bounded to impurity sites. The carrier spin strongly couples to the localized $d$ spins on Mn ions. The hopping of the carrier among Mn sites causes the ferromagnetic ordering of the localized spins through the double-exchange mechanism. The Curie temperature obtained by using conventional parameters agrees well with the experimental result.
\end{abstract}

75.50.Pp, 71.23.-k, 71.70.Gm

Typeset using REVTEX 
Although a considerable amount of experimental data has already been accumulated, the origin of the ferromagnetism in III-V-based diluted magnetic semiconductors (DMS's) has still not been clarified theoretically. Since the magnetic interaction between Mn ions has been shown to be antiferromagnetic in $n$-type $(\mathrm{In}, \mathrm{Mn}) \mathrm{As}$ or intentionally compensated $(\mathrm{Ga}, \mathrm{Mn}) \mathrm{As}$, the ferromagnetic interaction in III-V-based DMS's is most likely hole-induced. 0 The ferromagnetism may be caused by the effective exchange interaction between the delocalized carrier (or the hole originating from the shallow acceptor) and the localized magnetic moment ( $d$ spin) on the Mn ion. Matsukura et al. . referred to the Ruderman-Kittel-KasuyaYoshida (RKKY) interaction as the origin of the above ferromagnetism. However, the application of the RKKY interaction to this system, as pointed out by various authors 3 目 is somewhat questionable. The RKKY formula is applicable when the exchange energy $I S(\equiv I \times S)$ is small compared to Fermi energy, but this is not the case here because of

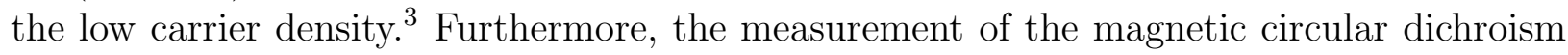
(MCD) spectrum of (Ga,Mn)As suggests that the holes play more active role in mediating the ferromagnetic exchange than traditional RKKY systems.6 To date, in regard to the origin of the above ferromagnetism, the double-exchange mechanism, 1 the double-resonance mechanism, 3 the Zener-model description, 7 and the self-consistent theory based on the CPA and mean-field theory $\mathrm{E}$ have been proposed.

In this letter, we apply the dynamical coherent potential approximation (dvnamical CPA) 910 to the carriers in $\mathrm{Ga}_{1-x} \mathrm{Mn}_{x}$ As described by a model Hamiltonian $H ; 12$ [13

$$
H=\sum_{m, n, \mu} \varepsilon_{m n} a_{m \mu}^{\dagger} a_{n \mu}+\sum_{m} U_{m},
$$

where $a_{m \mu}^{\dagger}\left(a_{m \mu}\right)$ creates (annihilates) a carrier (or $p$-hole) with spin $\mu$ at the $m$-th site. We treat $\mathrm{Ga}_{1-x} \mathrm{Mn}_{x}$ As as an alloy in which a mole fraction $x$ of Ga ions (denoted by symbol $A$ ) is replaced at random with $\mathrm{Mn}$ ions (denoted by symbol $M$ ). Hence $U_{m}$ is $E_{A} \sum_{\mu} a_{m \mu}^{\dagger} a_{m \mu}$ or $E_{M} \sum_{\mu} a_{m \mu}^{\dagger} a_{m \mu}-I \sum_{\mu \nu} a_{m \mu}^{\dagger} \boldsymbol{\sigma}_{\mu \nu} \cdot \mathbf{S}_{m} a_{m \nu}$ depending on whether the site $m$ is occupied by a Ga or a $\mathrm{Mn}$ ion. Here, $E_{A}$ and $E_{M}$ denote the spin-independent potentials for the Ga and the Mn ion, respectively; $-I \boldsymbol{\sigma} \cdot \mathbf{S}_{m}$ represents the $p$ - $d$ exchange interaction between the carrier and the localized ( $d$-) spin $\mathbf{S}_{m}$ of $\mathrm{Mn}$ at the site $m$. Hereafter, we set $E_{A} \equiv 0$ as the origin of the energy. A carrier moving in a DMS is subject to disordered potentials which arise not only from substitutional disorder but also from thermal fluctuations of the localized $(d-)$ spins. In the dynamical CPA, 11 the disordered potentials are taken into account in terms of the spin-dependent coherent potentials, $\Sigma_{\uparrow}$ and $\Sigma_{\downarrow}$, which are determined such that the effective scattering of a carrier at an arbitrarily chosen site embedded in the effective medium vanishes on average. The condition (the dynamical CPA condition) is given by

$$
\begin{aligned}
& (1-x) t_{\uparrow \uparrow}^{A}+x<t_{\uparrow \uparrow}^{M}>_{\mathrm{av}}=0, \\
& (1-x) t_{\downarrow \downarrow}^{A}+x<t_{\downarrow \downarrow}^{M}>_{\mathrm{av}}=0 .
\end{aligned}
$$

The multiple scattering of an $\uparrow(\downarrow)$-spin carrier by an $A$ and an $M$ ion embedded in the effective medium is represented by the $t$ matrix element $t_{\uparrow \uparrow}^{A}\left(t_{\downarrow \downarrow}^{A}\right)$ and $t_{\uparrow \uparrow}^{M}\left(t_{\downarrow \downarrow}^{M}\right)$, respectively. Note that in the above expression for $t_{\uparrow \uparrow}^{M}\left(t_{\downarrow \downarrow}^{M}\right)$, the spin flip processes are properly taken into account.11 The thermal average over the fluctuations of the localized spins is taken by assuming that they are subject to an effective field $h$, and denoted by $<>_{\mathrm{av}}$. In this work we assume the localized spins to be classical. Assuming a semicircular density of 
states(DOS) with the half-bandwidth $\Delta$ for the host valence band, we solved Eqs (2a) and (2b) simultaneously, and obtained the DOS per site $D_{\uparrow}(\omega)\left(D_{\downarrow}(\omega)\right)$ of the carrier with $\uparrow(\downarrow)$ -spin.

We assume $\Delta=2 \mathrm{eV}, I S / \Delta=-0.4$ and $E_{M} / \Delta=-0.3$ as parameters for $\mathrm{Ga}_{1-x} \mathrm{Mn}_{x} \mathrm{As}$; $E_{M}(<0)$ is the attractive potential acting on a hole due to a $\mathrm{Mn}^{2+}$ ion (see later discussion). In the diluted impurity limit $(x \rightarrow 0)$, the present model gives an impurity (acceptor) level at the energy of $E_{a}=-1.057 \Delta$. With increase in Mn concentration an impurity band forms, and for $x>0.02$ it merges into the host band which originates from the valence band of the host semiconductor. This result is consistent with the observed insulator- metal transition in $\mathrm{Ga}_{1-x} \mathrm{Mn}_{x}$ As at $x \sim 0.03$. 14 The result of the carrier DOS for $x=0.05$ is shown in Fig. 1(a) for various values of magnetization $\left\langle S_{z}\right\rangle_{\mathrm{av}} / S$. Note that the system with $x=0.05$ is metallic. The band tail arising from the magnetic impurity band is strongly affected by magnetization. With increase of the magnetization the lower edge of the DOS is slightly shifted to the lower energy and at the same time $D_{\uparrow}(\omega)$ is strongly suppressed in the band tail. We show the optical carrier spin polarization defined by $P(\omega)=\left(D_{\uparrow}(\omega)-D_{\downarrow}(\omega)\right) /\left(D_{\uparrow}(\omega)+D_{\downarrow}(\omega)\right)$ in Fig.1(b). In order to examine the exchange coupling of carriers with localized spins, we calculated the relative local DOS at Mn sites, $R(\omega)=\left(D_{\uparrow}^{\mathrm{M}}(\omega)+D_{\downarrow}^{\mathrm{M}}(\omega)\right) /\left(D_{\uparrow}(\omega)+D_{\downarrow}(\omega)\right)$, and depicted it in Fig.1(c). The result shows that a carrier at the band tail stays mainly at Mn sites $(R(\omega) \sim 0.6)$ in spite of small $x$. It should be noted that $R(\omega)$ is almost independent of $\left\langle S_{z}\right\rangle_{\text {av }}$. The result suggests that the change in $P(\omega)$ is mainly ascribed to the change at the magnetic ion sites.

In order to examine how strongly the carrier spin couples to the localized spins we calculate the carrier spin polarization $P(n) \equiv\left(n_{\downarrow}-n_{\uparrow}\right) /\left(n_{\downarrow}+n_{\uparrow}\right)$ as a function of the total carrier density $n$. Throughout this study, we assume that the carriers are degenerate. In Fig.2, we show the results of $P(n)$ for $x=0.01$ and $x=0.05$. When $x=0.01$, the impurity band is separated from the host band, so that carriers stay mainly at Mn sites as long as $n \lesssim x$. The carrier spin at a Mn site is expected to follow faithfully the fluctuations of the localized spin. This leads to the result that $P(n) \approx<S_{z}>_{\text {av }} / S$ for $n \lesssim x(=0.01)$, as shown in Fig.2(a). When $x=0.05$, the magnetic impurity band partly merges into the host band and the Fermi level $\left(\varepsilon_{F}\right)$ reaches the bottom of the host band at the carrier density $n_{1} \sim 0.035$. Accordingly, $P(n) \approx<S_{z}>_{\text {av }} / S$ for $n \lesssim n_{1}$ as shown in Fig.2(b). When $n \gtrsim n_{1}$, carriers with energy higher than $-\Delta$ seeps out of Mn site to itinerate among nonmagnetic sites.

We calculate the total energy as a function of $\left\langle S_{z}\right\rangle_{\text {av }}$ as

$$
E\left(<S_{z}>_{\text {av }}\right)=\int_{-\infty}^{\varepsilon_{F}} \omega\left[D_{\uparrow}(\omega)+D_{\downarrow}(\omega)\right] d \omega
$$

Note that $E\left(<S_{z}>_{\text {av }}\right)$ is the sum of the kinetic and exchange energy. We show in Fig.3(a) the energy difference between the ferromagnetic and the paramagnetic state as a function of $n$. Figure 3(a) reveals that the energy gain is approximately proportional to $\left(\frac{\leq S_{z}>\mathrm{av}}{S}\right)^{2}$ at a fixed $n$.

The free energy of the present model per site is given as

$$
F\left(<S_{z}>_{\mathrm{av}}\right)=E\left(<S_{z}>_{\mathrm{av}}\right)-T \mathcal{S}
$$

where the entropy term due to the localized spins is given by 


$$
\mathcal{S}=x k_{B} \log \sum_{S_{z}=-S}^{S} \exp \left(\frac{h S_{z}}{k_{B} T}\right)-x \frac{h}{T}<S_{z}>_{\mathrm{av}}
$$

The effective field $h$ is determined so as to minimize $F\left(<S_{z}>_{\text {av }}\right)$ through the condition $\frac{d}{d h} F\left(<S_{z}>_{\text {av }}\right)=0$. If we can expand $F\left(<S_{z}>_{\text {av }}\right)$ in terms of $\left(<S_{z}>_{\text {av }}\right)^{2}, T_{c}$ is determined as the temperature where the coefficient of $\left(\left\langle S_{z}\right\rangle_{\text {av }}\right)^{2}$ vanishes. While calculations for small magnetization accurate enough to estimate the coefficient is difficult, we observed from Fig.3(a) that $\left.E\left(<S_{z}>_{\text {av }}\right)-E(0)\right]$ is approximately proportional to $\left.\left(<S_{z}\right\rangle_{\text {av }}\right)^{2}$ up to full polarization. Therefore we fitted the data of $\left[E\left(<S_{z}>_{\text {av }}\right)-E(0)\right] / \Delta$ with $\left(<S_{z}>_{\text {av }} / S\right)^{2}=$ 0.4 and 1.0 to the expansion $\left.E\left(<S_{z}>_{\mathrm{av}}\right)-E(0)\right] / \Delta=-a\left(<S_{z}>_{\mathrm{av}} / S\right)^{2}+b\left(<S_{z}>_{\mathrm{av}}\right.$ $(S)^{4}$ and estimated $a$ and $b$ in actual calculation. Then $T_{c}$ is given by $k_{B} T_{c}=2 a / 3 x$. In Fig.3(b), the result of $T_{c}$ is depicted as a function of $n$ for $x=0.05$. It has been reported that ferromagnetism with Curie temperature $T_{c}=110 \mathrm{~K}$ is realized in $\mathrm{Ga}_{1-x} \mathrm{Mn}_{x} \mathrm{As}$ with $x=0.053$ when $n$ is $30 \%$ of the nominal concentration of Mn. The present result is in excellent agreement with the experimental result.

In order to obtain further insight into the mechanism of the carrier-induced ferromagnetism in DMS's, we consider the case that the exchange interaction is so strong that the carrier spins keep almost antiparallel to the fluctuating localized spins. We show the result for $I S / \Delta=-1.0$ and $E_{M} / \Delta=0$ in Fig. 4 for illustration. The magnetic impurity band is formed about the impurity level (marked 'A') and is separated from the host band. The total number of states in the impurity band per site is equal to $x$ irrespective to the value of $\left\langle S_{z}\right\rangle_{\text {av }} / S$. When $\left\langle S_{z}\right\rangle_{\mathrm{av}}=S$, all states in the impurity band are down-spin state, while when $\left\langle S_{z}\right\rangle_{\mathrm{av}}=0$ the impurity band is composed of the same number $(x / 2)$ of upand down-spin states. The ferromagnetic DOS extends over a wider energy range about the impurity level than does the paramagnetic DOS. Consequently the ferromagnetic state has lower energy than the paramagnetic state when $n$ is small. This result implies that the double-exchange mechanism 15 works in the magnetic impurity band to realize ferromagnetism. The energy gain increases first with the increase of $n$ and reaches a maximum value at $n \sim x / 2$. Then it decreases gradually and vanishes finally at $n \sim x$ as shown in Fig. 3(b). The same mechanism is expected to be operative in the case of $I S=-0.4 \Delta$ and $E_{\mathrm{M}}=-0.3 \Delta$ although the impurity band is not separated in this case. Therefore we conclude that the ferromagnetism in $\mathrm{Ga}_{1-x} \mathrm{Mn}_{x} \mathrm{As}$ with $x=0.05$ is caused by the doubleexchange mechanism in the band tail.

Here, we discuss the parameters and the model used in the present work. Since the width of the valence band is estimated to be about $4 \mathrm{eV}$ by band calculations, 16 , 17 we have taken $\Delta=2 \mathrm{eV}$. There are controversial discussions on the magnitude (even on the sign) of the exchange constant in III-V-based DMS's. Therefore, we have assumed that $I S / \Delta=-0.4$, which is a conventional value in II-VI-based DMS's. 22 Note that $\left|\frac{E_{M}+I S}{\Delta}\right|>0.5$ is the necessary condition for the existence of an impurity level. Accordingly, the attractive potential $E_{M}<0$ is needed to reproduce an impurity level, which is confirmed by corelevel photoemission experiment 18 Since the impurity level $E_{a}$ is given by $\frac{E_{a}}{\Delta}=\left(\frac{E_{M}+I S}{\Delta}\right)+$ $\frac{1}{4}\left(\frac{\Delta}{E_{M}+I S}\right)$ in the present model, we introduced the attractive potential $E_{M}=-0.3 \Delta$, which reproduces experimental value of the acceptor energy $0.113 \mathrm{eV}(=0.057 \Delta) .19$ We emphasize that the attractive potential strongly assists the occurrence of the ferromagnetism. If we take $E_{M} / \Delta=0$ together with $I S / \Delta=-0.4$, which corresponds to Mn doped II-VI DMS's, $T_{c}$ is 
calculated to be at highest $40 \mathrm{~K}$ for $x=0.05$. This is because the carrier in the II-VI-based DMS's spreads over many nonmagnetic sites, which reduces the effective exchange coupling. The result explains well why III-V-based DMS's such as (Ga,Mn)As exhibit appreciable ferromagnetism while II-VI-DMS's such as (Cd,Mn)Te and (Zn,Mn)Te exhibit only negligible ferromagnetism. To the contrary, if we take $E_{M} / \Delta=-0.6$ together with $I S / \Delta=-0.4$, the magnetic impurity band separates from the host band, and $T_{c}$ increases to $140 \mathrm{~K}$ for $x=0.05$. In the latter case, the carrier is semi-localized to a magnetic impurity site by the attractive potentials so that the double-exchange mechanism works efficiently.

In the present model, the carrier transfer energy $\left(\varepsilon_{m n}\right)$ between the Mn site and Ga site is assumed to be the same as that between Ga sites. That is, holes in (Ga,Mn)As are assumed to have mainly the As $4 p$ character which is originated from the valence-band maximum of the host GaAs, rather than Mn 3d character (although we actually treated only carriers on cation sites). Therefore the present model is in contrast to the double-exchange model based on the Mn 3d band. 1 Results of recent studies using the angle-resolved photoemission spectroscopy 20 and the band-structure calculation using the local-density-approximation with Coulomb interaction (the LDA $+\mathrm{U}$ method) 17 strongly support our assumption.

In summary, we have shown that the carriers in the band tail of $\mathrm{Ga}_{1-x} \mathrm{Mn}_{x}$ As have so large local-carrier-density on magnetic ion sites that the carrier spins well couples the localized spins fluctuating on the Mn ions. The double-exchange mechanism in the magnetic impurity band causes the ferromagnetism in the DMS. The attractive local potentials at impurity sites strongly assist the occurrence of the ferromagnetism in III-V-based DMS's.

\section{ACKNOWLEDGMENTS}

The authors would like to thank Professor Y. Kayanuma for informing them of the work of his group before publication. One of the authors (M. T.) is grateful to Professor W. Nolting for his instructive comments on the carrier-induced ferromagnetism. K. K. was

partially supported by Center for Science and Engineering Research, Research Institute of Aoyama Gakuin University. 


\section{REFERENCES}

* Tel/ Fax: +81-46-291-3107.

E-mail address: taka@gen.kanagawa-it.ac.jp.

${ }^{1}$ H. Ohno, J. Magn. Magn. Mater. 200, 110 (1999).

${ }^{2}$ F. Matsukura, H. Ohno, A. Shen, and Y. Sugawara, Phys. Rev. B 57, R2037 (1998).

${ }^{3}$ O. Sakai, S. Suzuki, and K. Nishizawa, J. Phys. Soc. Japan 70, 1105 (2001).

${ }^{4}$ H. Akai, Phys. Rev. Lett. 81, 3002 (1998).

${ }^{5}$ J. Inoue, S. Nonoyama, and H. Itoh, Phys. Rev. Lett. 85, 4610 (2000).

${ }^{6}$ B. Beschoten, P. A. Crowell, I. Malajovich, D. D. Awschalom, F. Matsukura, A. Shen, and H. Ohno, Phys. Rev. Lett. 83, 3073 (1999).

${ }^{7}$ T. Dietl, H. Ohno, F. Matsukura, J. Cibert, and D. Ferrand, Science 287, 1019 (2000).

${ }^{8}$ M. Yagi, K. Noba, and Y. Kayanuma, J. of Lumin. 94-95, 523 (2001).

${ }^{9}$ K. Kubo, J. Phys. Soc. Japan 36, 32 (1974).

${ }^{10}$ D. M. Edwards, A. C. M. Green, and K. Kubo, J. Phys. Condens.: Matter 11, 2791 (1999).

${ }^{11}$ M. Takahashi and K. Mitsui, Phys. Rev. B 54, 11298 (1996).

${ }^{12}$ M. Takahashi, Phys. Rev. B 60, 15858 (1999).

${ }^{13}$ M. Takahashi, J. Phys.: Condens. Matter 13, 3433 (2001).

${ }^{14}$ A. Oiwa, S. Katsumoto, A. Endo, M. Hirakawa, M. Iye, H. Ohno, F. Matsukura, A. Shen, and Y. Sugawara, Solid State Comm. 103, 209 (1997).

${ }^{15}$ C. Zener, Phys. Rev. 81, 403 (1951).

${ }^{16}$ T. Ogawa, M. Shirai, N. Suzuki, and I. Kitagawa, J. Magn. Magn. Mater. 196-197, 428 (1999).

17 J. H. Park, S. K. Kwon, and B. I. Min, Physica B 281 \& 282, 703 (2001).

${ }^{18}$ J. Okabayashi, A. Kimura, O. Rader, T. Mizokawa, A. Fujimori, T. Hayashi, and M. Tanaka, Phys. Rev. B 58, R4211 (1998).

${ }^{19}$ M. Linnarsson, E. Janzen, B. Monemar, M. Kleverman, and A. Thilderkvist, Phys. Rev. B 55, 6938 (1997).

${ }^{20}$ J. Okabayashi, A. Kimura, O. Rader, T. Mizokawa, A. Fujimori, T. Hayashi, and M. Tanaka, Phys. Rev. B 64, 125304 (2001). 


\section{FIGURES}

FIG. 1. (a) The Carrier (hole) density of states for $x=0.05$ are depicted for various values of magnetization. The solid and dotted lines represent $D_{\downarrow}(\omega)$ and $D_{\uparrow}(\omega)$, respectively. The arrow indicates the Fermi level for $n=x(=0.05)$, which is about $-0.95 \Delta$ and is almost independent of $\left\langle S_{z}>_{\mathrm{av}}\right.$. (b) The optical carrier spin polarization defined by $P(\omega)=\left[D_{\downarrow}(\omega)-D_{\uparrow}(\omega)\right] /\left[D_{\downarrow}(\omega)+D_{\uparrow}(\omega)\right]$. The impurity level $(\omega / \Delta=-1.057)$ is also indicated by a dot (symbol 'A'). (c) The ratio of the local DOS at Mn site to the total DOS, $R(\omega)=\left[D_{\uparrow}^{M}(\omega)+D_{\downarrow}^{M}(\omega)\right] /\left[D_{\uparrow}(\omega)+D_{\downarrow}(\omega)\right]$, for $<S_{z}>_{\mathrm{av}} / S=0.0,0.5$, and 1.0.

FIG. 2. The carrier spin polarization defined by $P(n)=\left(n_{\downarrow}-n_{\uparrow}\right) /\left(n_{\downarrow}+n_{\uparrow}\right)$ for various degrees of magnetization for $x=0.01$ (a) and $x=0.05$ (b). The arrows indicate $n=x$ and $n=n_{1} ; n_{1}$ is the carrier density where the Fermi level $\left(\varepsilon_{F}\right)$ reaches the bottom of the host band.

FIG. 3. (a) $\left[E\left(<S_{z}>_{\text {av }}\right)-E(0)\right] / \Delta$ is depicted for $x=0.05$ as a function of the carrier density

$n$, for various values of $\left(\left\langle S_{z}\right\rangle_{\text {av }} / S\right)^{2}$. (b) The Curie temperature $T_{c}$ calculated for $x=0.05$ as a function of $n$.

FIG. 4. The DOS of the magnetic impurity band in the case of $I S / \Delta=-1.0$ and $E_{M} / \Delta=0$. The thick, thin and dotted lines show the cases of $\left\langle S_{z}\right\rangle_{\text {av }} / S=1.0,0.5$ and 0.0 , respectively. The magnetic impurity level is dotted with symbol 'A'. 